FORMATION Formation emploi

Revue française de sciences sociales

107 | juillet-septembre 2009

La formation professionnelle en Amérique latine

\title{
Demandes de formation et formation pour le travail dans le secteur des services financiers en uruguay: biais de genre et évaluation par les compétences
}

Training requests and training for work in the financial-services sector: gender bias and skills assessment

Bildungserfordernisse und Berufsausbildung im Bereich der

Finanzdienstleistungen. Gender Bias und Beurteilung anhand von Kompetenzen Demandas de capacitación y formación para el trabajo en el sector de servicios financieros: sesgos de género y evaluación por competencias

\section{Mariela Quiñones Montoro}

Traducteur : Mireille Zangani

\section{(2) OpenEdition} Journals

Édition électronique

URL : http://journals.openedition.org/formationemploi/2013

DOI : 10.4000/formationemploi.2013

ISSN : 2107-0946

Éditeur

La Documentation française

Édition imprimée

Date de publication : 1 septembre 2009

Pagination : 57-72

ISSN : 0759-6340

Référence électronique

Mariela Quiñones Montoro, «Demandes de formation et formation pour le travail dans le secteur des services financiers en uruguay: biais de genre et évaluation par les compétences », Formation emploi [En ligne], 107 | juillet-septembre 2009, mis en ligne le 01 septembre 2011, consulté le 30 octobre 2020. URL : http://journals.openedition.org/formationemploi/2013 ; DOI : https://doi.org/10.4000/ formationemploi.2013 


\section{DOSSIER}

\section{Demandes de formation et formation pour le travail dans le secteur des services financiers en Uruguay: biais de genre et évaluation par les compétences}

Mariela Quiñones Montoro*

Si l'approche par les compétences favorise l'instauration de pratiques plus transparentes et universelles, elle est loin d'être neutre dans ses effets, particulièrement en ce qui concerne les différences de carrières entre femmes et hommes.

Soumis à de profondes transformations dues à la nécessité de s’adapter aux exigences du marché, le secteur des services financiers a été largement affecté au cours des dernières décennies par des changements rapides ; à savoir, une forte demande d'innovations technologiques - organisationnelles et de procédés -, et une force de travail de meilleure qualité.

Dès lors, un changement dans le paradigme de la qualification professionnelle apparaît dans le secteur. Ce changement, que nous analyserons dans cet article, vise à faciliter le développement des " compétences » des ressources humaines susceptibles de mieux contribuer à la capacité d'adaptation de l'entreprise et à son efficacité face à l'incertitude et au changement accéléré de l'environnement.
* Mariela Quiñones Montoro est docteur en sociologie de l'université autonome de Barcelone, enseignante et chercheure adjoint à temps plein au Département de sociologie de la faculté de sciences sociales et de la filière des relations professionnelles de la faculté de droit (Université de la République). Mail : Mariela@fcs.edu.uy. Elle a notamment publié : Quiñones M. et Supervielle M. (2005), "Xestión de Cultura Organizacional e prácticas de recursos humanos na Banca Española Multinacional" in Revista Galega de Economía, Año 14, n 1, Universidad Santiago de Compostela: Santiago de Compostela. Quiñones M. (2005), Demandas de capacitación y formación para el trabajo en el sector de servicios financieros- sesgos de género y evaluación por competencias (LC/L.2267-P), Santiago de Chile: Serie Mujer y Desarrollo n 61, Unidad Mujer y Desarrollo, CEPAL. 
On observe un mouvement qui peu à peu délaisse la spécialisation croissante de tâches et les systèmes d'apprentissage centrés sur le poste de travail (qui caractérisaient traditionnellement les faibles exigences en matière de qualification du secteur), au profit d'un modèle de travail basé sur le retour au " savoir-faire ", sur la capacité des individus à mobiliser et à développer des potentiels dans les situations concrètes de travail.

Ainsi, autre maillon dans cette grande chaîne d'effets articulés, ces nouvelles exigences en matière de travail ont largement influencé les conditions de fonctionnement du marché du travail grâce à un changement notable de la demande de travailleurs et, par conséquent, de qualifications ; cela s'est traduit par une force de travail jeune, possédant des niveaux élevés d'éducation et largement féminine, puisque dans la majorité des pays les femmes représentent la moitié des effectifs du secteur. Dès lors, le mode de fonctionnement de ces marchés diffère de leur mode de fonctionnement traditionnel, caractérisé par une force de travail masculine et par de faibles exigences en termes de diplômes.

Cet article se propose, en privilégiant l'approche par le genre, d'apporter quelques éléments analytiques et comparés sur les demandes de compétences et de formation pour le travail dans le secteur des services financiers. À cet effet, nous mobilons d'une part, des études visant à pointer le potentiel désintégrateur produit d'une distribution inégale des ressources (qu'il s'agisse de connaissances, relations, rémunérations, prestiges, etc..) - de ces processus de changement organisationnel (Calmon Arruda, 2000) ${ }^{1}$ et, d'autre part, des statistiques du marché du travail qui attestent, au cours des dernières décennies, d'un fort accroissement de la demande de force de travail féminine comme effet de ces changements,

Nous pensons que l'approche de la formation et du processus par lequel s'instaure un nouveau paradigme de qualification dans les entreprises financières, qui domine à l'heure actuelle, n’a pas été

\footnotetext{
${ }^{1}$ Processus de déqualification, déplacement, centralisation des ressources dans un groupe de travailleurs et d'exclusion sociale résultant de cette polarisation intra-organisationnelle qui accompagne ces transformations et qui se définit comme un processus de marginalisation et de segmentation.
}

évaluée du point de vue des résultats, des effets non désirés qui les accompagnent.

L'article se centrera donc sur les procédures institutionnelles, les représentations autour des significations des règles de conduite, sur les attentes, les préjugés et les systèmes de codification et d'interprétation qui émergent de la mise en œuvre de ce paradigme de la qualification basé sur les compétences. Ces éléments sont les expressions des cultures organisationnelles qui se conforment aux normes et fonctionnent comme des cadres de référence en orientant l'organisation des activités, le langage et les attitudes mises en jeu, les critères de mise en œuvre des outils de gestion, et influencent ainsi leurs résultats.

Précisons que cette étude fait appel, dans sa conception méthodologique, à l'examen de bases bibliographiques et de recherches sur le thème en Amérique latine. En complément, on exploitera les bases de données issues des enquêtes sur les ménages dans cinq pays (Argentine, Chili, Costa Rica, Salvador et Uruguay) et on approfondira la question en Uruguay grâce à une enquête représentative de la population bancaire (AEBU, 2001), et à des entretiens approfondis auprès d'informateurs qualifiés, principalement travailleurs (24) et responsables des ressources humaines (8 sur un total de 10 banques) $)^{2}$. Dans la partie centrale, consacrée à l'analyse méticuleuse du système de gestion par les compétences, on utilise une étude de cas d'une banque multinationale espagnole en Uruguay, qui procède aux évaluations de l'activité des salariés.

À partir de cette orientation et selon cette méthodologie, dans une première partie, l'article contextualise le secteur à partir des transformations qui, dans les dernières décennies, ont influé sur la demande du facteur travail. Dans une seconde partie, il aborde les principaux changements organisationnels à l'aune de deux politiques qui semblent avoir structuré la gestion des ressources humaines au cours des dernières décennies, les exigences en matière d'éducation et la formation pour le travail.

\footnotetext{
2 Signalons que dans le pays, comme dans le reste du continent, le secteur a été traversé par un fort processus de concentration et de prise de participation étrangère dans l'économie, ce qui a conduit à une large internationalisation; on observe de fortes tendances homogénéisantes dans la gestion des ressources humaines.
} 
Tableau 1

Répartition de l'emploi des services financiers selon le sexe et le pourcentage de femmes en Uruguay

\begin{tabular}{|l|c|c|c|c|}
\hline & Hommes & Femmes & Total & \% de femmes au total \\
\hline $\begin{array}{l}\text { A- Membres du gouvernement et dirigeants } \\
\text { d'entreprises publiques }\end{array}$ & 12.4 & 1.9 & 7.9 & 9.9 \\
\hline B- Universitaires & 7.8 & 7.6 & 7.7 & 41.6 \\
\hline $\begin{array}{l}\text { C- Techniciens et professionnels de niveau } \\
\text { moyen }\end{array}$ & 13.7 & 10.11 & 12.2 & 35.1 \\
\hline D- Employés de bureau & 57.2 & 69.1 & 62.3 & 47.0 \\
\hline E- Employés du secteur services et vendeurs & 0.0 & 6.0 & 7.0 & 92.9 \\
\hline $\begin{array}{l}\text { F- Opérateurs qualifiés arts mécaniques et } \\
\text { autres métiers }\end{array}$ & 0.0 & 0.0 & 0.0 & 0.0 \\
\hline $\begin{array}{l}\text { G- Opérateurs de maintenance et des } \\
\text { machines }\end{array}$ & 2.9 & 0.0 & 1.6 & 0.0 \\
\hline H- Travailleurs non qualifiés & 5.2 & 5.2 & 5.2 & 41.9 \\
\hline
\end{tabular}

Source : Enquête permanente ménages (Argentine, 2002). Enquête permanente ménages à buts multiples, juillet 2002 (Costa Rica) ; Enquête de caractérisation socio-économique CASEN, (Chili, 2000) ; Emploi, chômage et sous-emploi en zone urbaine (Équateur, décembre 2002) ; Enquête ménages à buts multiples (Salvador, juillet à décembre 2001) ; Enquête permanente ménages (Uruguay ; 2003).

Dans cette partie, on analysera plus précisément comment l'insertion des femmes s'opère dans ces processus de changement et sur la base de quelles exigences en matière d'éducation et de qualification.

Suite au constat de la présence ou de l'absence des femmes dans le secteur, l'étude comporte une troisième partie sur l'analyse de ce qui semble constituer le modèle guidant les niveaux de cette demande, le modèle de gestion par les compétences, substitut du paradigme taylorien antérieur centré sur l'évaluation de la tâche. La recherche que nous avons réalisée avec Supervielle (2003a) autour du système d'évaluation de l'activité d'une filiale uruguayenne (gérée depuis l'étranger) d'une banque multinationale espagnole, rendait compte de cette tendance. À cet égard, il serait pertinent ici de nous arrêter sur ses résultats en matière de gestion car ils suscitent la réflexion sur la façon dont, dans la mise en œuvre d'outils de gestion, interviennent des critères techniques et de rationalité substantive qui sont aussi influencés par les cultures entreprenariales et profes- sionnelles. Dans les processus de prise de décision, les routines, les résistances au changement, les inerties des procédures établies, les stéréotypes, etc., continuent à opérer... autant d'éléments qui doivent être pris en compte dans l'évaluation des résultats. Dès lors, nous examinons les possibles implications de ce modèle sur l'évolution des inégalités de genre.

Pour terminer, on récapitule les résultats les plus notables en conclusion. À cet égard, l'étude rend compte du fait que ces politiques agissent comme un déclencheur de nouveaux mécanismes d'inégalité dans le secteur, par exemple, l'accès différencié aux diverses modalités de formation proposées par l'entreprise, les critères très stéréotypés dans la sélection des compétences requises pour accéder à des postes hiérarchiques et/ou à de meilleures rémunérations. Après avoir rendu compte de ces résultats, nous formulons quelques préconisations afin que les différents acteurs impliqués dans la thématique réorientent les politiques de formation pour les adultes depuis les différentes institutions compétentes. 


\section{SYSTÈME FINANCIER EN AMÉRIQUE LATINE : LES TRANSFORMATIONS ET LEUR IMPACT SUR LA DEMANDE DU FACTEUR TRAVAIL}

L'activité financière traditionnelle s'est caractérisée par un marché interne avec une forte stabilité de l'emploi et des avantages sociaux multiples. Conséquence de cette logique, le système bancaire, pilier de l'activité au cours des dernières décennies, a été marqué par différents mouvements étroitement liés : l'augmentation de l'ancienneté au détriment de la qualification effective, un marché "profil professionnel d'activité » spécifique, la spécialisation, la stabilité dans l'emploi.

La concentration du personnel dans les activités de vente correspond aux exigences de bas niveau de qualification (Larangeira, 2001; Quinones, 2004). Dans de nombreux cas, ce qui caractérisait les cadres intermédiaires était davantage leur capacité à intégrer les critères de l'entreprise et à les imposer au personnel qu'une qualification supérieure à leurs collègues. Ceci dénotait un travail d'inspiration fortement taylorienne, standardisé, pouvant être segmenté, avec des systèmes bureaucratiques et hiérarchiques de traitement de l'information.

Cette conjonction d'éléments aurait eu une incidence sur la configuration d'un marché interne également très rigide, caractérisé par la prééminence d'une force de travail masculine de bas niveau de qualification et par la stabilité dans l'emploi. Intégrés sur cette base, les employés disposaient de systèmes d'apprentissage sur le poste de travail qui leur permettaient d'acquérir de l'expérience dans la plupart des aspects du travail et d'amples connaissances de base. On parlait de carrières d'une durée comprise entre trente et quarante ans. La femme est nettement exclue du secteur, puisque quand on en employait une, on l'affectait à un poste subalterne et il s'agissait rarement du démarrage d'une carrière professionnelle dans la banque.

Le type de réorganisation qui a suivi ce modèle s'est inscrit dans la recherche d'une flexibilité accrue qui impliquait d'accompagner les changements décrits précédemment. La nécessité d'une capacité d'adaptation rapide à l'instabilité croissante du marché s'est essentiellement traduite par la primauté accordée aux tâches liées à l'activité commerciale, par le fait que le personnel a été libéré de tâches auparavant typiques des employés de niveaux inférieurs (principalement par rapport à la vente), par une intégration croissante des processus de travail et, comme corollaire, par une plus grande polyvalence ${ }^{3}$.

Ainsi, le marché interne des services financiers commence à privilégier une force de travail jeune, avec de hauts niveaux d'éducation; ce qui s'est traduit par une nette augmentation de la demande de main-d'œuvre féminine. Par exemple, en étudiant l'entrée de personnel possédant un niveau supérieur complet, et même un diplôme de 3e cycle, uniquement dans le secteur bancaire, Lanrangeira (2001) parle, pour le Brésil, d'un accroissement de 28 à $38 \%$ dans la période comprise entre 1994 et $1999^{4}$. Ces variations sont comparables aux données d'autres pays de la région comme l'Uruguay, où le pourcentage dans la même période a varié de 28 à $41 \%$ ou au Costa Rica où, pour la période 19912000, il a crû de 25 à $54 \%$.

\footnotetext{
${ }^{3}$ Dans le sens de Zarifian (1999, p. 12) comme «extension de la superficie de la compétence de la personne ».

${ }^{4}$ Larangeira (2001) à partir des données de la Federaçao Brasileira das Associaçoes de Banco (1996).

${ }^{5}$ Données issues de l'Enquête de l'Association bancaire d'Uruguay (AEBU), département de sociologie (Faculté de sciences sociales - Université de la République) (2001). Dans ces données, on n’a pas identifié de travailleurs ou travailleuses possédant des niveaux d'éducation inférieurs au second cycle complet (secondaire), ces derniers étant ceux qui représentent, dans cette catégorie, que ce soit pour le suivi d'études universitaires complètes ou incomplètes ou pour avoir reçu n'importe quel type d'enseignement supérieur para-universitaire (école normale ou professorat), $80 \%$ de la population interviewée. Pour le Costa Rica, le traitement de l'Enquête à buts multiples de 2002 révèle que 69 \% de la population de l'ensemble de l'activité financière (non uniquement bancaire) a accompli un cycle d'études secondaires complet. Signalons que la réplique de l'enquête faite en Uruguay cette année confirme et enregistre une accentuation de cette tendance.
} 


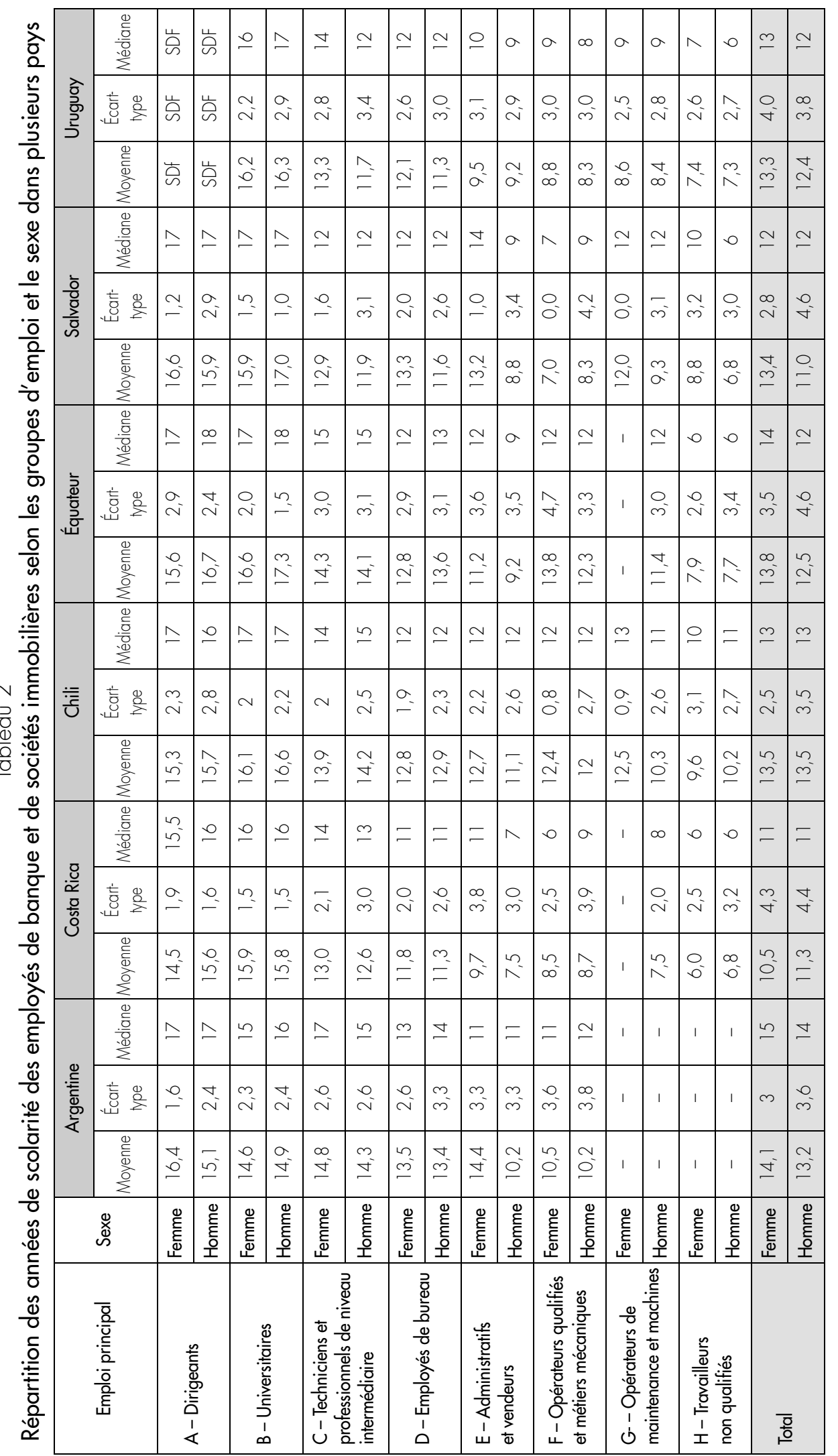

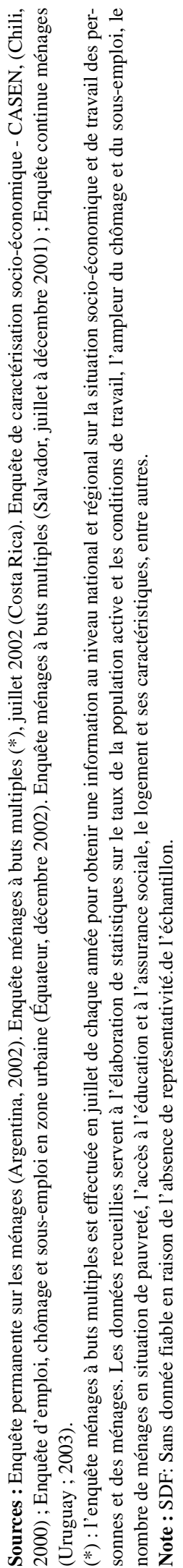




\section{LES CARACTÉRISTIQUES DE LA DEMANDE D'ÉDUCATION ET LEUR INCIDENCE SUR LES INÉGALITÉS DE GENRE}

Considérant ces nouvelles exigences d'utilisation et d'incorporation de la force de travail d'un côté, et la forte croissance des niveaux d'éducation formelle des femmes qui façonnent l'offre, de l'autre, sur la base de quelles exigences en matière d'éducation et de qualification les femmes s'insèrent-elles dans ces processus de changement?

Quant à la question de savoir s'il existe une demande différentielle liée au sexe, aucune preuve ne corrobore cette hypothèse. L'ouverture du marché du travail financier aux femmes n'a pas engendré de conditions d'emploi différentes pour des postes égaux. L'analyse des profils éducatifs évalués selon la moyenne et la médiane de la variable « années d'études dans l'enseignement formel » par grandes catégories de la variable «emploi principal », conduit à mettre en évidence l'accroissement global des exigences éducatives pour tous les travailleurs et travailleuses du secteur. Les tableaux 1 et 2 fournissent cette information désagrégée, de même que l'information selon le « sexe ».

Cependant, l'analyse de la demande de niveaux éducatifs relève plusieurs résultats. D’un côté, les exigences éducatives, élevées dans tout le secteur, croissent à mesure que l'on gravit l'échelle professionnelle hiérarchique. La moyenne des années d'études enregistre, dans tous les pays, une différence de 4 à 6 ans entre les catégories à bas niveau de qualification - vendeurs ou travailleurs des services et/ou de bureau - et les postes de direction, professionnels et techniciens possédant une qualification élevée. On conclut dès lors que les femmes $a$ priori ne seraient ni par les études, ni par leur condition naturelle exclues des postes hiérarchiques. Dès lors, il est possible de différencier clairement les mécanismes d'inégalité à l'œuvre dans le modèle taylorien antérieur, comportant des normes très asymétriques et anti-féminines centrées sur l'exclusion a priori des femmes d'emplois et/ou d'occupations, et le modèle de qualification actuellement à l'œuvre, dans lequel est privilégié l'apport du travailleur en connaissance et où le genre apparaît comme quelque chose d'autonome par rapport à son développement, et non comme le mécanisme (visible) de discrimination.

À la suite de ces constats, et en considérant quelques études de trajectoires professionnelles dans le secteur (p.e. Quinones, 2005), nous nous proposons d'examiner plus attentivement ce que nous appelons présence et absence des femmes dans le secteur des services financiers et les processus sociaux sur lesquels elles ont pu se fonder, afin d'analyser l'existence de modèles d'inégalités persistantes.

La première conclusion qui ressort de cet examen est nette; si nous pouvons parler, comme Carrasquer (1996,) d'une certaine «normalisation» de la présence des femmes, toutes les études révèlent que, à mesure que leur participation augmente, leur présence dans la hiérarchie organisationnelle diminue ; ce qui nous incite à penser que l'égalisation de l'offre éducative pour les hommes et les femmes et leur insertion, également plus égalitaire au sein des structures professionnelles du secteur, ne l'auraient pas emporté aujourd'hui sur la ségrégation verticale des postes de travail. À partir de ces résultats, nous avons commencé à accorder de l'importance à ces hypothèses qui, comme les travaux de Mauro (2005), s'orientent vers la reconstruction d'itinéraires et de cheminements professionnels (trajectoires) à partir de l'étude des conditionnements structurels, institutionnels et/ou individuels qui pourraient être à l'origine de ces processus ${ }^{6}$.

Ainsi nous commençons à mesurer l'importance actuelle prise par des pratiques telles que la « formation interne $»^{7}$ et « l'évaluation de la performance » en tant

\footnotetext{
${ }^{6}$ L'étude des trajectoires professionnelles a ouvert tout un champ de possibilités pour la compréhension du monde du travail. Non seulement elles rendent visibles les opportunités offertes à une période déterminée aux hommes et aux femmes, mais aussi les relations entre les changements socio-économiques et culturels, d'un côté, et les transformations des subjectivités personnelles et sociales, de l'autre.

${ }^{7}$ Concernant une telle demande de qualification lors du recrutement, un des gérants interviewés précise : "Nous ne pouvons pas demander à l'université qu'elle se substitue à nous comme formateurs; il est logique que les élèves ne connaissent pas les derniers fonds de placement du marché ». Mais si les universités ne forment pas les salariés en matière de connaissances techniques spécifiques, on attend d'elles qu'elles leur apportent une formation «sur des valeurs comme l'effort ou l'auto-développement, l'acceptation des risques".
} 
que pratiques qui orientent le développement de nouvelles dynamiques liées à l'accroissement du secteur, les exigences en matière d'études supérieures étant une condition nécessaire mais pas suffisante pour évaluer la performance du travailleur ou son affectation sur un poste de travail.

Dès lors, nous proposons quelques hypothèses moins explorées : par exemple, à conditions de rémunérations identiques, les opportunités de formation continue pourraient constituer un obstacle à l'égal accès des femmes aux postes à responsabilité. En d'autres termes, ces pratiques pourraient agir comme déclencheur de nouveaux mécanismes d'inégalité dans le secteur.

Selon l'information disponible, cette hypothèse serait confirmée par les formes de participation des hommes et des femmes aux cours de perfectionnement et/ou de formation à la gestion, surtout dans le domaine financier, notoirement différenciées, et qui mettent en évidence de fortes divergences dans les critères des choix formatifs entre les sexes ${ }^{8}$. Selon les responsables des ressources humaines, le type

La culture générale, dans ce contexte, apparaît comme une condition nécessaire pour s'insérer dans un monde organisationnel complexe et pour s'adapter à l'évolution du secteur, c'est-à-dire, les aptitudes à l'apprentissage continu et l'adaptabilité pour occuper différents postes de travail ou différentes fonctions d'un même poste. À ce sujet, un autre cadre déclare que : «dans notre banque, chaque employé bénéficiera d'un plan individuel de formation qui permettra son intégration à l'effectif, un système d'évaluation, prime individuelle, et tout cela lié à un plan d'entreprise pour les cadres ».

${ }^{8}$ Par rapport à l'importance, pour le revenu, de la nécessité d'une formation dispensée par l'entreprise, l'information fournie par l'enquête sur les salariés du secteur bancaire en Uruguay (AEBU, 2001) est révélateur à cet égard. On note ainsi que 85 \% des salariés du secteur bancaire du pays ont suivi une formation interne. Cependant, si nous examinons la qualité des cours dispensés à l'aune de leur durée, nous observons que cette politique est l'objet d'une forte segmentation. $45 \%$ de la population bancaire a suivi des cours au sein de ce que nous pourrions appeler une activité " d'actualisation », car il s'agit de formations qui n'excèdent pas 20 heures. $46 \%$ a suivi des formations dans le cadre d'une politique de formation qui, par sa durée, pourrait être destinée à améliorer la spécialisation et/ou la reconversion - formations d'une durée de 20 à 120 heures - et seulement $9 \%$ a suivi des formations de plus de 120 heures, soit ceux qui pourraient s'intégrer dans une politique de formation professionnelle. En nous référant toujours à cette enquête, les cours les plus mentionnés par les personnes interrogées ont été, dans l'ordre : informatique, gestion bancaire, vente, langues, marketing et commerce extérieur. Les cours d'informatique et de gestion bancaire étant dominants au sein des cours de formation professionnelle de la banque. Soulignons que nous n'avons pas noté de différences significatives entre les formations et leur mise en œuvre en fonction du sexe de la personne interrogée. d'emploi occupé étant une condition pour progresser dans la carrière, beaucoup de femmes peuvent éprouver des difficultés pour accéder à des cours de formation en dehors des horaires de travail ou encore à la mobilité géographique (considérée comme action formative) ; cela expliquerait qu'on les trouve aux niveaux de qualification peu élevés et, dans de nombreux cas, sur des postes peu protégés. Cet avis n’est pas partagé par les salariées, qui disent accéder aujourd'hui plus qu'hier aux cours de formation.

Face à ce désaccord, qui se fait jour aussi par rapport aux statistiques d'accès aux formations dans le secteur (AEBU-DS, 2001), nous pourrions affirmer qu'il s'agit d'un cas d'émergence, parmi les gestionnaires, d'une sorte de fausse attente mais qui a des conséquences concrètes sur les salariées au moment où ces gestionnaires sont enclins à défavoriser les femmes dans l'attribution de formations longues (cours de formation professionnelle). C'est pourquoi ces attentes agissent comme des prophéties pouvant s'accomplir dans le futur par la mise en place de procédures qui viseraient la réalisation de ces attentes...

Le second argument fort qui ressort des entretiens et qui confirme les interprétations mentionnées dans le paragraphe précédent, est l'évocation de l'existence de « compétences naturelles » des femmes pour exercer des emplois et/ou fonctions déterminés. En règle générale, il est banal de recueillir des opinions selon lesquelles les perspectives d'insertion des femmes sont meilleures dans les domaines tels que l'administration, la vente, le télémarketing ou sur des postes de secrétaires, standardistes, etc., au sein desquels les exigences en matière de "compétences sociales", également appelées «savoir-être » (collaboration, capacité relationnelle, dévouement, etc.) se fondent sur des compétences reconnues comme « féminines ».

À cet égard, il ne s'agit pas d'interroger empiriquement l'importance de ces capacités, mais de savoir comment elles sont situées et valorisées. Nous pensons, suivant en cela Zarifian (1999), que si l'on identifie ces compétences comme "qualités personnelles » - ce que la notion de « savoir-être » tend à faire - on place le débat sur les compétences en dehors d'un quelconque point de vue basé sur la professionnalité, pour en faire une évaluation des compétences personnelles. Se produit alors un 
chevauchement de situations; si on analyse les compétences comme « disposition », " responsabilité » ou « communication », on devrait le faire au sein des compétences professionnelles - qui agissent de façon autonome par rapport à des situations professionnelles, et non "en général »; cela permettrait de déterminer comment elles peuvent s'acquérir et de quels moyens disposent les entreprises pour les développer ou détecter leur carence le cas échéant. C’est uniquement sous cet aspect qu'il est possible d'intégrer les compétences dans un débat autour des niveaux de professionnalité du secteur.

En définitive, les tenants de ces opinions ne font que réduire le thème à une question de point de vue personnel, quand ce qui est en jeu c'est la reconnaissance sociale de ces compétences qui permet de leur attribuer les niveaux de professionnalité correspondant aux comportements standards exigés aujourd'hui par les entreprises. L'effet pervers de ces croyances est que le fait de les exprimer ainsi peut opérer à nouveau comme des "prophéties autoréalisatrices ». Ainsi, considérer que les femmes sont mieux dotées pour des empois et fonctions précis conduit, d'un côté, à ce que les femmes soient envisagées uniquement pour ces emplois et/ou fonctions et, d'un autre côté, à ce qu'elles en viennent à croire qu'elles ne sont capables que de faire cela, ce qui expliquerait qu'elles puissent fournir moins d'efforts et moins s'investir pour accéder à d'autres emplois ou se former pour accéder à d'autres fonctions. Ainsi la femme construirait son identité professionnelle et son sens de "la carrière » dans le travail sur ces compétences « stéréotypées féminines ».

Ces constats et opinions nous ont incités à poursuivre la recherche. Mais cette fois, sur le terrain, grâce à des observations systématiques permettant de délimiter cet espace des demandes de compétences, de connaître leur complexité ${ }^{9}$; il s'agit de rompre avec des explications linéaires selon lesquelles derrière tout problème il y a un manque de qualification, une condition naturelle ou une absence de disposition. Nous analysons cette problématique dans la partie suivante.

\footnotetext{
${ }^{9}$ Pour une classification exhaustive des compétences professionnelles, $c f$. Martens (1996).
}

\section{CONTENUS DE LA QUALIFICATION QUI AGISSENT SUR LA SEGMENTATION PAR GENRE AU SEIN DU SECTEUR}

Selon les données utilisées, un mouvement progressif vers l'évaluation et la gestion par les compétences disponibles parmi le personnel se serait opéré dans le secteur. Les nouveaux contenus des entretiens de sélection, de même que le développement d'évaluations de performance, les réunions assidues autour des responsables de secteurs ou les cours de formation interne seraient destinés à favoriser un contact intense et même une relation personnelle entre employés et responsables de leur promotion, de telles pratiques s'appuyant sur un modèle de développement d'une logique compétences sur laquelle, insistent les gestionnaires, les titres académiques jouent un rôle de signal pour une première sélection parmi les candidats au recrutement (voir encadré 1).

\section{Les évaluations de la performance : la transition vers un modèle basé sur les compétences}

Le premier élément pris en compte dans l'analyse des résultats est que ces facteurs diffèrent selon que le personnel évaluateur a une position hiérarchique (cadre) ou non (non cadre). Voir à cet effet le tableau 3.

Nous constatons que si la distinction proposée entre cadres et non cadres est largement acceptée dans le secteur, dans quelle mesure ces dimensions standardisées et majoritairement reconnues par les entreprises comme une distinction simplement formelle, se rapprochent-elles de «catégories » hiérarchiques substantives dans la subjectivité des employés de banque $^{10}$ ? On propose alors de passer de l'analyse de

\footnotetext{
10 On utilise le terme “catégorie” au même sens que la Grounded Théory, qui reprend la traduction aristotélicienne du concept "impératif catégorique”. L’approche par la Grounded theory est supposée favoriser l'innovation scientifique par une méthode d'enracinement de l'analyse dans les données de terrain. Il s'agit d'une approche inductive par laquelle l'immersion dans les données empiriques sert de point de départ au développement d'une théorie sur un phénomène et par laquelle le chercheur conserve toujours le lien d'évidence avec les données. Cette théorie est définie en opposition aux approches hipotético-déductives dans lesquelle les chercheurs partent de postulats a priori pour déduire des explications des
} phénomènes ( $c f$. Barney Glaser et Anselm Strauss, 1967). 


\section{Encadré ]}

\section{L'évaluation de la performance dans le secteur bancaire}

Cette banque d'Uruguay a présenté un cadre d'évaluation de l'exercice de l'emploi à appliquer à des emplois de cadres définis grâce à l'échelle hiérarchique. L'outil varie selon que les évalués ont du personnel sous leur ordre ou non (échelle pour cadres et échelle pour non cadres). À cet effet, l'entreprise utilise deux outils, deux matrices à double entrée qui, sur le plan horizontal, utilisent une série de facteurs (qui sur un plan technique peuvent être considérés comme variables) et qui varient légèrement entre les deux grilles, et sur le plan vertical, une série de valeurs: insuffisant, acceptable, bon, très bon et excellent, qui vont de 1 à 5 . Chaque case de la matrice contient une brève définition indicative pour l'évaluateur.

Les grilles d'évaluation comportent deux facteurs communs au personnel cadre et non cadre. L'évaluateur doit remplir pour chaque facteur une case et il fait ensuite la moyenne, une note qui résume l'évaluation. Parallèlement, il argumente brièvement, pour chaque facteur, le choix de chaque case, et s'il le désire, utilise la partie " observations générales » pour noter une quelconque impression globale sur l'évalué.

\section{Facteurs d'évaluation de cadres et de non cadres}

\begin{tabular}{|c|c|}
\hline Facteurs pour les cadres & Facteurs pour les non-cadres \\
\hline Activité professionnelle & Activité professionnelle \\
\hline Leadership & Qualité du travail \\
\hline Organisation et planification & Initiative \\
\hline Relations interpersonnelles & Relations interpersonnelles \\
\hline
\end{tabular}

Ceux qui évaluent sont les cadres immédiatement supérieurs (hiérarchie $n+1$ ), qui, dans certains cas, sont des cadres argentins et dans d'autres des uruguayens. Toutes les évaluations sont surpervisées par un évaluateur de niveau supérieur, d'origine argentine.

Méthodologie : en premier lieu, l'information a été réordonnée en fonction des évaluateurs, en distinguant les évaluations de cadres des évaluations de non-cadres et le sexe de l'évalué. L'objectif était de reconstruire le système de catégories grâce auxquelles on évalue le personnel. La reconstruction des systèmes de codes tente d'étudier la consistance du monde social bancaire en fonction de la répétition de codes des divers évaluateurs ; à partir des différences par rapport à la position de cadre ou non-cadre, on cherche à détecter les différences qui proviennent du sexe de l'évaluateur. On note que tous les évaluateurs sont des hommes. 
Tableau 3

\section{Capacités requises dans le travail de cadre et de non-cadre}

\begin{tabular}{|c|c|c|}
\hline \multicolumn{3}{|c|}{ Capacités requises pour le travail de cadre } \\
\hline Capacités académiques & $\begin{array}{c}\text { Capacités de développement } \\
\text { personnel }\end{array}$ & Capacités sociales et relationnelles \\
\hline $\begin{array}{l}\text { Capacité analytique } \\
\text { Capacité d'apprentissage } \\
\text { Capacité de compréhension } \\
\text { Capacité de communication } \\
\text { Connaissances } \\
\text { Intérêt pour la formation }\end{array}$ & $\begin{array}{l}\text { Anticipation } \\
\text { Confiance en soi } \\
\text { Capacité de travail } \\
\text { Collaboration } \\
\text { Fiabilité } \\
\text { Implication } \\
\text { Motivation } \\
\text { Intérêt pour des tâches diverses } \\
\text { Envie de s'améliorer } \\
\text { Leadership } \\
\text { Organisation } \\
\text { Précision } \\
\text { Responsabilité }\end{array}$ & $\begin{array}{l}\text { Motivation pour fidéliser la clientèle } \\
\text { Travail en équipe } \\
\text { Esprit de service } \\
\text { Apporter des améliorations } \\
\text { dans le travail } \\
\text { Capacité de planification }\end{array}$ \\
\hline \multicolumn{3}{|c|}{ Capacités requises pour le travail de non-cadre } \\
\hline Capacités académiques & $\begin{array}{c}\text { Capacités de développement } \\
\text { personnel }\end{array}$ & $\begin{array}{c}\text { Capacités de communication } \\
\text { et sociales }\end{array}$ \\
\hline $\begin{array}{l}\text { Capacité d'apprentissage } \\
\text { Capacité de compréhension } \\
\text { Capacité de communication } \\
\text { Connaissances } \\
\text { Intérêt pour la formation }\end{array}$ & $\begin{array}{l}\text { Adaptation au poste de travail } \\
\text { Auto-exigence } \\
\text { Capacité à se dépasser } \\
\text { Fiabilité } \\
\text { Investissement } \\
\text { Potentiel de développement } \\
\text { Disponibilité } \\
\text { Aptitudes } \\
\text { Motivation à s'améliorer } \\
\text { Exprimer de l'inquiétude } \\
\text { Précision } \\
\text { Rapidité } \\
\text { Responsabilité }\end{array}$ & $\begin{array}{l}\text { Aptitude participative } \\
\text { Collaboration } \\
\text { Partager des savoirs avec les collègues } \\
\text { D'accord pour assumer de nouvelles } \\
\text { tâches } \\
\text { Apporter des améliorations dans } \\
\text { le travail } \\
\text { Intérêt pour des tâches diverses } \\
\text { Motivation pour fidéliser les clients } \\
\text { Travail d'équipe } \\
\text { Esprit de service }\end{array}$ \\
\hline
\end{tabular}

Source : Supervielle y Quiñones (2003).

la simple information numérique à l'analyse des distinctions a priori, qui instaure l'outil comme « catégories conceptualisantes »; c'est-à-dire en termes de révélateurs du sens que les individus donnent à leurs actions ou au monde qui les entoure. À cet égard, les distinctions deviendraient des distinctions substantives du « monde social bancaire $»^{11}$.
${ }^{11}$ Nous entendons par " monde social », suivant Shibutani : « un univers de réponses réciproques régularisées, (...) une arène où existe une sorte d'organisation (...) une zone culturelle où les frontières ne sont délimitées ni par un territoire
.......
Pour savoir si c'est le cas, la recherche nécessite des méthodologies d'analyse qualitative (analyse de contenu) afin de donner du contenu à ces distinctions qui sont confrontées aux résultats numériques des évaluations. Les découvertes sont notables. En premier lieu, à l'examen des données quantitatives, l'étude rend compte que les évaluateurs (toujours des

ni par leur appartenance formelle mais par les limites d'une communication efficace », cité par Anslem Srauss dans La trame de la négociation: sociologie qualitative et interactionnisme, Logiques sociales, L'Harmattan. 
cadres), en évaluant d'autres cadres, s'auto-évaluent systématiquement avec des notes plus élevées que lorsqu'ils évaluent le personnel non cadre. Ce qui révélerait une sorte de fermeture autopoétique ${ }^{12} \mathrm{du}$ personnel cadre et une nette séparation avec le reste du personnel. Parallèlement, en analysant les commentaires rédigés par les évaluateurs lors de la notation de chaque facteur ${ }^{13}$, on observe que dans les " évaluations des cadres » il y a une prise en compte beaucoup plus individualisée de l'évalué que dans les « évaluations des non-cadres ». Dans ce dernier cas, l'évaluation est moins précise et plus distante. Essentiellement, elle révèle que quand on évalue un cadre, on le fait sur des «individualités ", tandis que l'évaluation des non-cadres est réalisée en référence à ce que l'on appelle « l'individu-masse ». De même, dans la mesure où tandis que les cadres s'autoévaluent en appelant cela " compétences », chez les non-cadres il y a une prise en compte beaucoup plus grande des tâches puisque l'on évalue si leur réalisation est correcte. ${ }^{14}$

Ceci conduit à construire une typologie qui distingue «évaluations individuelles/évaluations individus-masse » et "évaluations sur le travail/ évaluations sur les tâches ». Ces constats sont suffisamment étayés pour que l'on attribue la distinction personnel cadre et personnel non cadre, plus qu'à une simple distinction méthologique à la coexistence de deux cultures de travail qui s'expliquent par l'organisation même du monde social bancaire (Quinones et Supervielle, 2005) ; une culture que nous appelons, par analogie, "taylorienne », qui considère le travail essentiellement en fonction des tâches considérées isolément et l'individu en tant qu'individu-masse, et une autre culture, avec une vision plus holiste et réflexive du travail. Ces deux cultures, non explicites, impliqueraient des métacritères d'évaluation très différents. Dans un cas, la

${ }^{12}$ Voir Luhmann (1998). En bref, il s'agit de la propriété d'un système à se produire, se maintenir et se définir lui-même.

${ }^{13}$ Notons que ce commentaire est presque de rigueur dans l'évaluation comme une façon de justifier la qualification vis-à-vis de l'évalué et d'apparaître comme un mécanisme de transparence de la qualification.

${ }^{14}$ Ce point s'articule avec le précédent puisque l'évaluation par les compétences est beaucoup plus individualisante tandis que l'évaluation de tâches collectives implique une prise en compte plus collective de l'évalué réalisation correcte des tâches prévaudrait ; dans la seconde, ce seraient les compétences ${ }^{15}$.

La seconde réflexion provient de la nécessité de nous interroger sur l'utilisation que ces évaluations de l'exercice de l'activité font de ce que l'on appelle «l'objectif compétences ». N'oublions pas que l'origine historique du modèle des compétences repose justement sur la thèse du besoin de requalification des travailleurs dans le nouveau modèle économique et que pour certains, il doit être envisagé en termes de dépassement du modèle taylorien de qualification (Zarifian, 1999 ; Hirata, 1996) ${ }^{16}$. Selon les conclusions de notre étude, cette rupture n'est pas aussi nette et, même si elle est en recul, la culture taylorienne continue à dominer aujourd'hui dans le monde social bancaire. Au point que c'est sur ce critère qu'est définie l'existence de la microculture des cadres. Du point de vue du genre, cette réflexion est aussi d'un grand apport dans la mesure où aujourd'hui, dans la banque, les femmes sont beaucoup plus représentées sur des postes subalternes, évalués à l'aune de critères purement tayloriens.

\section{Compétences directes : évaluation par compétences et biais de genre}

Le second apport est issu d'une seconde étude que nous avons réalisée sur ce même cas, qui aborde les évaluations par une approche de genre (Quinones, 2005). Nous recherchons les catégories qui permettent au secteur bancaire de justifier des distinctions telles que travail et compétences « féminines et/ou masculines ». Notre thèse est que, dans la mesure où ces outils s'insèrent dans des structures organisationnelles plus ou moins solides, comme les évaluations sont réaffirmées par les divisions hiérarchiques, des

\footnotetext{
${ }^{15}$ Ceci étant, il est important de signaler que la culture " taylorienne » est aujourd'hui minoritaire et en recul, mais elle était majoritaire dans le secteur. Nombreux sont les actuels salarié-e-s à avoir été formé-e-s dans cette culture, et non seulement elle transparaît dans les évaluations mais elle oriente aussi leurs résultats.

${ }^{16}$ En termes généraux, cette contradiction naît essentiellement du fait que la qualification se référerait au poste de travail, au salaire, aux tâches, tandis que la compétence aurait trait à la subjectivité, la multifonctionnalité et l'imprécision. L'individu serait alors rémunéré pour sa “capacité", pour sa performance et non pour l'emploi qu'il occupe. Ainsi, le modèle compétences permettrait, dans ce sens, de disposer des outils rhétoriques nécessaires pour opérer une rupture avec la notion de poste de travail, avec l'encadrement et la rémunération élevée, imposant à cet égard une forte composante individualisante.
} 
dynamiques bien connues depuis la métaphore déjà évoquée des prophéties qui s'accomplissent fatalement peuvent à nouveau être alimentées ; comme les compétences se définissent à partir des rôles attribués et/ou assumés par les hommes et les femmes, on finit par attribuer des caractéristiques et des capacités à des emplois en fonction des caractéristiques de leurs occupants.

À nouveau, plusieurs résultats ressortent. On observe que, tandis que pour les femmes les qualifications qui contribuent le mieux à une bonne évaluation sont celles que nous appelons "académiques » et «de travail en équipe », pour les hommes, les commentaires ont trait aux qualifications que nous appelons « de développement personnel ». Même si l'on ne doit pas considérer ces qualifications comme s'excluant les unes les autres, l'accent mis sur les unes pour évaluer les femmes et sur les autres pour évaluer les hommes implique, sinon une hiérarchie, du moins l’idée implicite de complémentarité entre les sexes.

Cela signifie que, même si les deux sexes peuvent être évalués positivement dans n'importe quel domaine de qualifications et compétences, les évaluations hautes des femmes sont centrées sur les compétences académiques et de travail en équipe : " entretenir des relations cordiales ", "favoriser la communication ", " résoudre des problèmes ", « communiquer», etc., compétences qui, selon certaines opinions, seraient plus spécialement féminines, tandis que les qualifications des hommes ont davantage trait à des compétences de développement personnel, comme «capacité de travail», " collaboration », " responsabilité », " atteindre des objectifs », " précis et fiable », "disponibilité ». Jusqu'à présent, tout concorde pour que l'on puisse dire que aussi bien les hommes que les femmes possèdent, avec une intensité différente, les « compétences d'encadrement » déjà analysées.

Un second constat, complémentaire au précédent, a trait aux compétences communes qui sont évaluées positivement à la fois chez les hommes et les femmes. À cet égard, l'appel à une compétence commune a-t-il la même connotation s'il se réfère à des personnes de sexe différent? Cela n'est pas toujours le cas. Par exemple, au cours de l'analyse, on rend compte de compétences comme «capacité de planification » mais qui, dans les évaluations, sont attachées à des interprétations très différentes d'un emploi à un autre. On qualifie positivement une employée à l'aune de ce facteur car « elle fait preuve d'une organisation adéquate mais au jour le jour », alors que ce sens correspond peu à l'idée forte du "savoir-faire » qui suppose de "planifier le travail de la section ». De ces exemples, on conclut que des compétences peuvent varier de sens selon que l'on évalue le travail exécuté par les individus de l'un ou l'autre sexe.

En outre, il apparaît pertinent d'analyser l'importance prise, dans le discours sur les exigences en matière de compétences d'encadrement, par l'idée du dirigeant comme "gestionnaire de connaissances ». Comme l'entreprise commence à être envisagée comme un système ouvert, la référence à un monde extérieur et un autre intérieur tend à devenir prégnante dans l'organisation. Cela correspondrait à l'organisation des compétences d'encadrement, pour autant qu'on fasse la distinction entre les compétences qui seraient précieuses dans la mesure où elles contribuent au maintien de l'environnement interne de l'organisation, et les compétences qui projettent la personne et, à travers elle, l'organisation, vers l'extérieur. Dans la littérature entreprenariale, cette distinction se reflète dans la séparation entre deux types de compétences stratégiques : « ex-stratégiques et in-stratégiques ». Les compétences d'encadrement « ex-stratégiques » étant celles nécessaires pour obtenir de bons résultats économiques et qui comprennent des capacités comme la perception et la résolution de problèmes, la gestion de ressources, l'orientation vers le client et la construction de réseaux de relations efficaces ; compétences qui, dans l'étude mentionnée, seraient davantage associées aux hommes. À l'inverse, les compétences d'encadrement «instratégiques » sont définies comme nécessaires pour développer les emplois et augmenter l'engagement et la confiance dans l'entreprise. Elles sont liées à la capacité à diriger et au leadership et parmi elles on trouve la communication, l'empathie, la délégation, le coaching et le travail d'équipe; compétences qui, selon les évaluations analysées, seraient davantage attribuées aux femmes.

La conclusion de l'étude est que, selon ces catégories substantives qui ordonnent le monde social bancaire, les femmes disposeraient de compétences propres 
relatives à des emplois de direction, mais même ainsi elles n’auraient pas développé «l'efficacité personnelle » et la "projection ex-stratégique », compétences essentielles pour être affectées sur ces postes. Pour les décrire, la littérature se réfère à la fonction de direction qui intègre la capacité à allier ces dimensions, à concevoir des stratégies susceptibles de produire de la valeur économique (Cardona, 2002).

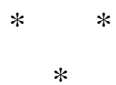

Tout au long de l'article, nous avons vu comment, au moment où le monde financier se trouve soumis à de forts changements, la demande de qualifications devient elle aussi chaque jour plus complexe. Et ce dans la mesure où le concept même de qualification se complexifie en accompagnant les mutations qui se produisent dans son environnement.

Nous avons vu comment aux compétences de base, conjugées à des exigences de hauts niveaux éducatifs dans le secteur, s'ajoute le besoin de connaissances spécifiques soumises à certaines caractéristiques individuelles et sociales (compétences) qui permettent aux salarié-e-s d'accéder à ces connaissances et de les développer, et d'être capables de les gérer dans le travail, conçu chaque jour davantage comme la résolution de problèmes, la capacité de se confronter à des contenus inédits, le cas échéant. Ces transformations peuvent engendrer des fractures en fonction de l'appartenance des salarié-e-s à différents groupes professionnels, mais un référentiel d'exigences s'est imposé pour tout le personnel employé aujourd'hui dans le secteur financier.

Mais même si cela témoigne d'une évolution institutionnelle vers un modèle unique, homogène et systémique de gestion des ressources humaines, qui réduit les différences intra-organisationnelles, paradoxalement, on observe comment les distinctions intraindividuelles augmentent en matière de qualifications, de trajectoires et d'espaces formatifs des travailleurs et quels sont leurs effets sur les types d'inégalité sociale dans le secteur. C'est donc autour de cette dimension inhérente au paradigme « des compétences » que notre étude s’est proposée de réfléchir, sur les effets de la mise en œuvre de ce modèle sur les inégalités de genre et les formes de discrimination présentes aujourd'hui dans le secteur. Ainsi, nous observons l'évolution de la demande de hauts niveaux d'éducation, les exigences de formation, le développement de systèmes d'évaluation destinés à accroître des compétences mais aussi à en détecter les manques.

Ainsi, nous avons vu que même si ce ne sont pas les différences scolaires qui contribuent le plus fortement à la segmentation et à la reproduction des inégalités dans le secteur bancaire, l'augmentation démesurée des exigences en matières éducative engendre une situation qui agit comme déclencheur d'autres mécanismes sociaux producteurs d'inégalité présents dans l'entreprise.

En effet, les différences scolaires augmentent l'écart scolaire par l'accès à des formations professionnelles et même à des formations soutenues par l'entreprise ou encore par la reconnaissance de compétences requises (celles-ci étant le plus souvent associées aux possibilités de promotion), cette dynamique tendant à créer de nouvelles inégalités.

Dans ce panorama, nous n'avons pas noté de différences significatives dans l'accès à la formation selon le sexe, ou bien selon le niveau scolaire initial, ni à catégorie égale ou à partir d'initiatives individuelles ; cependant, nous avons constaté que les femmes évoluent dans une aire de formation très différente de celle des hommes. Nous pensons donc que la dynamique de la formation dans le secteur bancaire fonctionne selon un constat ancien, celui du poids important qu'exercent les facteurs structurels et culturels sur les caractéristiques personnelles des formés dans la création d'inégalités dans l'entreprise, celui que certains auteurs évoquent en référence à la formation sur le développement d'autres inégalités, « inégalités supplémentaires » en termes d'augmentation salariales, de possibilités de construire des carrières internes auxquelles les hommes déclarent en général accéder plus fréquemment après une formation (Gaudrey, 1982).

Dans ce contexte, nous considérons que les apports de la sociologie sont importants quant à la connaissance des effets des pratiques de gestion des ressources humaines mises en œuvre par les entreprises du secteur bancaire. Au sein de ces entreprises, il est important de souligner que même si l'approche par les compétences progresse de façon importante 
grâce à l'instauration de pratiques plus transparentes et universelles, elles sont loin d'être neutres dans leurs effets, particulièrement en ce qui concerne les questions de genre.

Les témoignages et études de cas dans le secteur financier présentés ici mettent en évidence que, en essayant de définir des standards de comportement, la gestion par les compétences implique une logique très inique Puisque, au-delà de l'atteinte ou non de l'objectivité dans l'évaluation, elle ne prend pas en compte les facteurs (surtout culturels) qui agissent sur la définition même des concepts que l'on tente de mesurer par l'évaluation de la performance. En occultant les différences dans les choix formatifs des hommes et des femmes, leurs opportunités d'accès et d'évolution, la gestion par les compétences fonctionne comme une prophétie qui fatalement s'accomplit, engendrant un autre mécanisme reproducteur d'inégalité.

Ces résultats de recherche nous amènent à conclure que, sous le règne du paradigme dominant de l'organisation inspirée du taylorisme, la discrimination de genre s'est opérée directement sur le marché de l'emploi, à deux niveaux : à l'entrée, c'est-à-dire que le critère discriminatoire pré-existait - les femmes étaient absentes du secteur - et dans la carrière, où elles étaient uniquement recrutées pour des emplois précis et où elles étaient rarement promues. À l'inverse, s'agissant de l'évolution du travail dans le secteur au cours des dernières années, avec l'introduction de la gestion des ressources humaines et, avec elle, la substitution du paradigme de la qualification par le modèle des compétences, la discrimination ne s'opère pas à l'entrée mais par rapport aux compétences requises.

Dans la mesure où cette hypothèse est pertinente, elle est intéressante à plusieurs titres. En premier lieu, elle nous indique que la discrimination dans le modèle taylorien était de type asymétrique, antiféminin, tandis que ça n'était pas nécessairement le cas dans le modèle post-taylorien: la discrimination pouvant être plus neutre. Mais d'un autre côté, dans le premier cas, la discrimination était « externe »; elle opérait sur des emplois et des activités précis dont les femmes étaient a priori exclues. Dans le second cas, elle est « interne », opère au cœur du travail et, par conséquent, est nettement plus forte et plus présente, ce qui génère des discriminations beaucoup plus subtiles mais aussi plus ataviques dans leur fonctionnement réel.

Le paradoxe est que les évaluations par les compétences qui visent à détecter l'existence ou la carence de capacités - objectif clairement recherché - pourraient contribuer à entériner une division des activités attribuées à chaque sexe, de leur espace, carrière, rémunération et autres aspects liés au travail et à reproduire l'inégalité, comme l'ont montré les recherches évoquées. Le traitement de cette thématique est significatif puisque les institutions de formation professionnelle ont privilégié cet objectif comme instrument pour développer de meilleures opportunités d'égalité pour les femmes.

Pour terminer, tous ces constats nous ont conduit à adopter une vision différente de la formation basée sur le développement de compétences par rapport à la vision institutionnelle. Selon nous, l'approche par les compétences se veut une approche sur la professionnalité. Dans ce sens, il est important que l'entreprise reconnaisse au préalable quelles compétences sont requises pour des fonctions précises, mais aussi comment elles peuvent être acquises et de quels moyens elle dispose pour les développer. Nous pensons que ce moyen est la formation.

C'est pourquoi nous préconisons que dans les conditions actuelles la formation soit envisagée comme une instance de réflexion de tous les acteurs impliqués - travailleurs, chefs d'entreprises, formateurs, etc., lui conférant ainsi une plus grande rationalité et une démocratisation dans les décisions relatives à son domaine. La formation doit permettre d'accompagner et de suivre les transformations culturelles dans les organisations.

Plus particulièrement, nous pensons qu'il est nécessaire que les institutions s'emparent de manière réflexive de la façon de faire face aux exigences du monde productif et des résultats que doit produire une tâche liée à la culture dans laquelle elle sera imbriquée.

Traduit de l'espagnol au français par Mireille Zangani (Céreq), avec l'aimable collaboration de l'auteur. 


\section{Bibliographie}

Calmon Arruda M. de C. (2000), "Qualificação Versus Competência”, Boletín Cinterfor $n^{\circ} 149$, mayo-agosto de 2000, Montevideo; Organización Internacional del Trabajo, CINTERFOR.

Cardona P. (2002), Las Claves del talento. La influencia del liderazgo en el desarrollo del capital humano, España, Empresa Activa.

Carrasquer P. (1996), El Empleo femenino en el sector financiero en España: entre la diferencia y la desigualdad, Barcelona, Columna.

Deluiz N. (1994), "Formação do trabalhador em contexto de mudança tecnológica, Boletim Técnico do Senac, Rio de Janeiro, vol. 20, ${ }^{\circ}$ 1, jan./abr.

Gaudrey N. (1982), « Hommes et femmes au travail : inégalités, différences, identités », Paris L'Harmattan, voir C. Dubar G. Podevin (1990) Formation et promotion en France depuis vingt ans. Bref-Cereq.

Goux D. et Maurin E. (1997), « Les entreprises, les salariés et la formation continue" Économie et statistiques $\mathrm{n}^{\circ} 306$, in C. Dubar et Ch. Gadea La promotion sociale en France. Lille, Presses Universitaires du Septentrion.

Heller L. (2001), «El empleo femenino en los noventa ¿nuevos escenarios, nuevas ocupaciones ? " el caso argentino ». En Aguirre R. et Batthyány K. Coord. (2001), Trabajo, género y ciudadanía en los países del Cono Sur. Montevideo OIT-Cinterfor, AUGM, Udelar.

Hirata H. (1996), "Da polarização das qualificações ao modelo da competência”, en Ferretti C. J. et al. Novas tecnologias, trabalho e educação: um debate multidisciplinar. Petrópolis: Vozes. pp. 128-142.

Hola. E. et Todaro R. (1992), Los Mecanismos del Poder: Hombres y Mujeres en la Empresa Moderna. Santiago de Chile: Centro de Estudios de la Mujer ; Grupo Editor Latinoamericano.
Larangeira S. (2001), « Reestructuraçäo em bancos e Telecomunicaçoes no Brasil: excluidos e beneficiarios », en Aguirre R. y Batthyány K. Coord. Trabajo, género y ciudadanía en los países del Cono Sur. OITCinterfor, AUGM, Udelar. Montevideo.

Luhmann N. (1998), Complejidad y modernidad: de la unidad a la diferencia. Madrid, Editorial Trotta.

Martínez J. (2004), Costa Rica: Inserción laboral de las mujeres en los servicios financieros - Buenas y no tan buenas noticias (LC/L 2295-P), Santiago de Chile: Serie Mujer y Desarrollo nº 62, Unidad Mujer y Desarrollo, CEPAL.

Mauro A. (2004), Trayectorias profesionales de las mujeres en el sector de servicios financieros de Chile, (LC/L. 2177-P/E), Santiago de Chile: Serie Mujer y Desarrollo ${ }^{\circ} 59$, Unidad Mujer y Desarrollo, CEPAL.

Mertens L. (1996), Competencia laboral. Sistemas surgimiento y modelos, Montevideo, Cinterfor/OIT.

Quiñones M. y Supervielle M. (2005), “Xestión de Cultura Organizacional e prácticas de recursos humanos na Banca Española Multinacional”, en Revista Galega de Economía, Año 14 ; n 1, Universidad Santiago de Compostela: Santiago de Compostela, ISSN 1132-2799.

Quiñones M. (2005), Demandas de capacitación y formación para el trabajo en el sector de servicios financieros- sesgos de género y evaluación por competencias (LC/L.2267-P), Santiago de Chile: Serie Mujer y Desarrollo $\mathrm{n}^{\circ}$ 61, Unidad Mujer y Desarrollo, CEPAL.

Quinones (2004), Globalización de las relaciones laborales en el sector financiero. De la problemática de la difusión a la comunicación, Tesis Doctoral, Barcelona, Universitat Autònoma de Barcelona.

Rico Ma. Nieves (1996), Formación de Recursos Humanos Femeninos: Prioridad del crecimiento y de 
la equidad. Serie Mujer y Desarrollo ${ }^{\circ}$ 15, (LC/ L.947-P/E), Santiago de Chile, CEPAL.

Sainsaulieu R. (1991), «Les "Effets” Formation en Entreprise » chap. 4 du Traité des Sciences et Techniques de Formation (1999) sous la direction de Philippe Carré et Pierre Caspar, Dunod.

Sainsaulieu R. (1987), Sociologie de l'organisation et de l'entreprise. FNSP et Dalloz Ed. Presses.

Santana M. (2003), Transformações da identidade de trabalhadores no setor financeiro: estudos de caso nos bancos estatais em Sergipe. Mimeo. Río de Janeiro, IUPERJ. Disponible sur:

http://www.iuperj.br/Lusofonia/papers/

Maria\%20Helena\%20Santana\%20Cruz.pdf

Schkolnik M., Araos C., Machado F. (2005), Certificación por competencias como parte del sistema de protección social: la experiencia de países desarrol- lados y lineamientos para América Latina. Serie Políticas Sociales $n^{\circ} 113$, Santiago de Chile: CEPAL (LC/L 2438P).

Supervielle M. (2001), « Notas sobre la profesionalización del empleo en la Banca ", en Evaluación de desempeño (antecedentes, informe técnico y propuestas de debate), Comisión Representativa Discount Bank Latin American, AEBU, febrero de 2001.

Supervielle M. y Quiñones M. (2003), El revés de la evaluación de desempeño, Montevideo, FCS, Udelar, $\mathrm{DI} / 33$.

Zarifian P. (1999), Mutación de los sistemas productivos y competencias profesionales: la producción industrial de servicio. El modelo de la competencia y sus consecuencias sobre el trabajo y los oficios profesionales, Montevideo, Organización Internacional del Trabajo (Cinterfor/OIT).

\section{Résumé}

\section{Demandes de formation ef formation pour le travail dans le secteur des services financiers en Uruguay: biais de genre et évaluation par les compétences}

\section{Mariela Quiñones Montoro}

L'article aborde les changements qui sont intervenus dans le paradigme dominant de qualification en lien avec son impact sur les inégalités sociales de genre. La thèse est que dans la mesure où l'on parle de compétences destinées à évaluer l'abstrait - c'est-à-dire la capacité de mobilisation du savoir-faire - les changements ont de grandes conséquences sociologiques, dès lors que l'on discute des hypothèses sur lesquelles sont construites les normes d'inégalité du modèle taylorien antérieur. Pour rendre compte de ce changement, on étudie la catégorisation des compétences comme mécanisme de production de l'inégalité. À cet égard, l'article pose la question de l'activité de définition des compétences, soulignant la responsabilité sociale du sociologue dans la divulgation et la reconstruction de cette réalité.

Mots clés

Division sexuelle du travail, formation professionnelle, banque, Amérique du Sud, Uruguay Journal of Economic Literature: J 24 